\title{
EMERGING DRUG RESISTANT STREPTOCOCCAL ISOLATES FROM SKIN AND SOFT TISSUE INFECTIONS
}

\author{
Bhawna Sharma1, Rushika Saksena², Manisha Jain³, Rajni Gaind ${ }^{4}$
}

${ }_{1}^{1}$ Assistant Professor, Department of Microbiology, Vardhman Mahavir Medical College and Safdarjung Hospital, Delhi, India. ${ }^{2}$ Specialist, Department of Microbiology, Vardhman Mahavir Medical College and Safdarjung Hospital, Delhi, India. ${ }^{3}$ Assistant Professor, Department of Microbiology, Vardhman Mahavir Medical College and Safdarjung Hospital, Delhi, India. ${ }_{4}^{4}$ Senior Consultant and Professor, Department of Microbiology, Vardhman Mahavir Medical College and Safdarjung Hospital, Delhi, India.

\section{BACKGROUND}

\section{ABSTRACT}

Streptococcal species are the cause of a variety of skin and soft tissue infections (SSTIs) some of which are severe and can be lifethreatening. There are currently 74 species under the genus Streptococcus. Streptococcus pyogenes is the most pathogenic bacterium in the genus Streptococcus. We wanted to assess pyogenic potential of different species Streptococcus and their drug susceptibility patterns.

\section{METHODS}

This is a retrospective descriptive study done from Jan 2017 to Nov 2017. Total enrolled pus samples were 3300. The samples were processed conventionally and antibiotic sensitivity testing (AST) of all isolates was performed by Kirby Bauer's disc diffusion method as per CLSI guidelines.

\section{RESULTS}

$4.63 \%$ were culture positive for streptococcal isolates. Among streptococcal isolates, 128/153 (83.66\%) were Enterococcus species, 12/153 (7.84\%) were Streptococcus pyogenes and 10/153 (6.53\%) were Streptococcus agalactiae and 3/153 (1.96\%) were Streptococcus pneumoniae. For all Streptococcal isolates (153), 64\% (102/160) were male patients and 36\% (58/160) were female patients. Drug resistance is increasing for all the streptococcal species, and resistance to even high-end drugs like vancomycin was seen to be around 7.5\% in Enterococcus spp. Almost one third of the isolates of Streptococcus pyogenes were resistant to Erythromycin and Gentamicin. No resistance was seen to Linezolid in any of the Streptococcal isolates.

\section{CONCLUSIONS}

Streptococcus species such as S. pyogenes, S. agalactiae and Streptococcus pneumoniae are seen to be increasing in SSTIs. Bacterial isolates exhibited high to moderate levels of resistance against different classes of antibiotics. A continuous inspection should be carried out to monitor the susceptibility of these pathogens and chose appropriate regimens both for prophylaxis and treatment of surgical wound infections. There is a need to create a viable antibiotic policy and draft guidelines to prevent or reduce undirected use of antibiotics, and conserve their effectiveness for better patient management. Consistent dialogue between the microbiology department and the surgeons is required for management of such cases.

HOW TO CITE THIS ARTICLE: Sharma B, Saksena R, Jain M, et al. Emerging drug resistant streptococcal isolates from skin and soft tissue infections. J. Evolution Med. Dent. Sci. 2019;8(14):1131-1134, DOI: 10.14260/jemds/2019/251

\section{BACKGROUND}

Streptococcal species are the cause of a variety of skin and soft tissue infections (SSTIs) some of which are severe and can be life-threatening. There are currently 74 species under the genus Streptococcus. ${ }^{1}$ Streptococcus pyogenesis the most pathogenic bacterium in the genus Streptococcus; the agent that causes bacterial pharyngitis, impetigo, scarlet fever, rheumatic fever and a host of other infections including severe invasive diseases. ${ }^{2}$ It is implicated in a wide variety of SSTIs including impetigo, erysipelas, cellulitis, necrotizing fasciitis, myositis and myonecrosis. ${ }^{3}$ The past two decades

\section{'Financial or Other Competing Interest': None.}

Submission 12-02-2019, Peer Review 23-03-2019,

Acceptance 29-03-2019, Published 08-04-2019.

Corresponding Author:

Dr. Manisha Jain,

House No. 88,

Vivekanand Puri,

Sarai Rohilla,

New Delhi, India.

E-mail: manisha_jain29@yahoo.com

DOI: $10.14260 /$ jemds $/ 2019 / 251$ have also witnessed a resurgence of several infective syndromes of S. pyogenes, most notably necrotizing skin and soft tissue infections and scarlet fever. ${ }^{4}$ Streptococcus agalactiae is a gram-positive coccus belonging to group B of the Lancefield classification. ${ }^{5}$

Today, Group B Streptococcus (GBS) colonizes gastrointestinal tract and genitourinary tract of about $30 \%$ of healthy individuals, without any symptoms. ${ }^{6,7}$ During the past three decades there have been reports of increasingly invasive infections in adults unrelated to pregnancy, although arthritis and osteomyelitis were still considered rare. ${ }^{8} \mathrm{~A}$ recent multi-centre study of 171 patients with invasive GBS infections in non-pregnant adults has reported SSTIs in 31\% of cases. ${ }^{9}$

Streptococcus pneumoniae commonly and asymptomatically colonizes the upper respiratory tract of children and is a major cause of respiratory and meningeal infections. SSTIs constitute around $2.2 \%$ of all pneumococcal infections where a breakdown of the normal mucosal barrier plays a major role in the development of bacteraemia and other soft tissue infection. ${ }^{10}$ Besides, haemolytic streptococci, Enterococcus spp. are being increasingly isolated from SSTIs. ${ }^{11}$ Enterococci are generally known to cause urinary, 
bloodstream and gastrointestinal infections. However, the increasing use of cephalosporins has contributed to emergence of Enterococci as etiological agents of SSTIs especially after trauma and surgery. ${ }^{12}$

The inadvertent use of antibiotics leading to emergence of drug resistant pathogenesis a great healthcare challenge. Although Penicillin remains the drug of choice for Group A and Group B Streptococci, resistance to macrolides, lincosamides and fluoroquinolones has emerged. ${ }^{13}$ Similarly, incidence of difficult to treat Vancomycin resistant Enterococci (VRE) too is on the rise. ${ }^{14}$

Thus, the present study was aimed to assess pyogenic potential of different species of Streptococcus and their drug susceptibility patterns from patients with pus and/or wound discharge.

\section{METHODS}

\section{Study Design}

This is a retrospective descriptive study conducted at the department of microbiology in a tertiary care hospital of northern India from January 2017 to November 2017.

\section{Sampling Methods}

Microbiology database was screened for all Streptococcal isolates from pus, wound discharge and tissue samples collected from patients with SSTIs. A total number of 3300 pus samples were obtained for aerobic culture and sensitivity from different In-patient department (IPD) \& Outpatient department (OPD) of our hospital. Pus samples were collected with sterile disposable cotton swabs and aspirates in syringe and were transported and processed in the microbiology laboratory immediately.

\section{Sample Processing}

They were inoculated on to $5 \%$ sheep Blood agar (BA), MacConkey agar (MA) and Brain heart infusion broth. Direct microscopy was performed by gram stain examination of the samples. All available clinical details of patients were noted. Culture plates were incubated at $37^{\circ} \mathrm{C}$ for 24 to $48 \mathrm{hrs}$. under aerobic condition. After incubation, identification of bacteria from positive cultures was done using standard phenotypic methods. All Streptococcal isolates were confirmed by conventional and serological tests. The antibiotic sensitivity testing (AST) of all isolates was performed by disk diffusion method on Muller Hinton agar and interpreted as per CLSI guidelines and classified as sensitive and resistant (CLSI 2017).[15] Antimicrobial susceptibility testing was performed against the following antibiotics: ampicillin $(30 \mu \mathrm{g})$, gentamicin (10 $\mu \mathrm{g}$ and $120 \mu \mathrm{g})$, cefoxitin $(30 \mu \mathrm{g})$, linezolid (30 $\mu \mathrm{g})$, vancomycin $(30 \mu \mathrm{g})$, ciprofloxacin $(5 \mu \mathrm{g})$, erythromycin $(10 \mu \mathrm{g})$, clindamycin $(2 \mu \mathrm{g})$, Trimethoprim/Sulfamethoxazole $(1.25 \mu \mathrm{g} / 23.75 \mu \mathrm{g})$. Escherichia coli ATCC 25922 and Staphylococcus aureus ATCC 25923 were used for quality control. All the culture media, biochemical media and antibiotics used were obtained from Hi Media.

\section{Statistical Analysis}

Results obtained were entered in the excel sheet and analyzed by using MS Excel, 2007 version. The percentage of sensitivity and resistance was calculated for all the organisms.

\section{RESULTS}

A total of 3300 samples were received during the study period from January 2017 to November 2017, out of which $153(4.63 \%)$ were culture positive for streptococcal isolates. Among streptococcal isolates 128/153 (83.66\%) were Enterococcus species, 12/153 (7.84\%) were Streptococcus pyogenes, 10/153 (6.53\%) were Streptococcus agalactiae and $3 / 153(1.96 \%)$ were Streptococcus pneumoniae.

\begin{tabular}{|c|c|c|c|}
\hline Antibiotic & \% R & \%IS & \%S \\
\hline Ampicillin & 63.4 & 00 & 36.6 \\
\hline $\begin{array}{c}\text { Gentamicin High } \\
\text { Level (120) }\end{array}$ & 44.3 & 00 & 55.7 \\
\hline Erythromycin & 70.3 & 14.1 & 15.6 \\
\hline Ciprofloxacin & 46 & 24 & 30 \\
\hline Linezolid & 00 & 00 & 100 \\
\hline Vancomycin & 7.5 & 00 & 92.5 \\
\hline Chloramphenicol & 16 & 4.5 & 79.5 \\
\hline Table 1. Antimicrobial Profile of Enterococcus Species \\
(n=128)
\end{tabular}

\begin{tabular}{|c|c|c|c|}
\hline Antibiotic Name & $\% \mathrm{R}$ & $\%$ IS & $\% \mathrm{~S}$ \\
\hline Ampicillin & 00 & 00 & 100 \\
\hline Gentamicin & 33.33 & 00 & 66.67 \\
\hline Clindamycin & 00 & 00 & 100 \\
\hline Erythromycin & 33.33 & 00 & 66.67 \\
\hline Linezolid & 00 & 00 & 100 \\
\hline Vancomycin & 00 & 00 & 100 \\
\hline \multicolumn{4}{|c|}{$\begin{array}{l}\text { Table 2. Antimicrobial Profile of Streptococcus Pyogenes } \\
(n=12)\end{array}$} \\
\hline
\end{tabular}

\begin{tabular}{|c|c|c|c|}
\hline Antibiotic Name & \% R & \% IS & \% S \\
\hline Penicillin & 00 & 00 & 100 \\
\hline Ampicillin & 00 & 00 & 100 \\
\hline Cefoxitin & 20 & 20 & 60 \\
\hline Gentamicin & 40 & 20 & 40 \\
\hline Clindamycin & 30 & 00 & 70 \\
\hline Erythromycin & 20 & 10 & 70 \\
\hline Linezolid & 00 & 00 & 100 \\
\hline Vancomycin & 00 & 00 & 100 \\
\hline Chloramphenicol & 40 & 10 & 50 \\
\hline Tetracycline & 100 & 00 & 00 \\
\hline Table 3. Antimicrobial Profile of Streptococcus Agalactiae \\
(n=10) \\
\hline
\end{tabular}

In 128 isolates of Enterococcus species 60\% were from In-patients ward (IPD) and $40 \%$ were from outpatient department (OPD). For Streptococcus pyogenes, Streptococcus pneumoniae and Streptococcus agalactiae 90\% were from IPD and 10\% were from OPD. The most common specialities were General Surgery followed by Orthopaedics for all kinds of isolates. For all Streptococcal isolates (153), $64 \%$ (98/153) were male patients and 36\% (55/153) were female patients. The level of resistance was very high in Enterococcus species as is shown in Table 1 . Almost $7.5 \%$ of the isolates were resistant to high end drugs like Vancomycin. Even in Streptococcus pyogenes resistance to commonly used antimicrobials such as Erythromycin and Gentamicin was high (up to 33.33\%) (Table 2). The Antimicrobial susceptibility of Streptococcus agalactiae is shown in Table 3. In case of Streptococcus pneumoniae it is difficult to comment on the level of resistance as the number of isolates was less but out of three isolates seen in our study one isolate was resistant to Erythromycin. 


\section{DISCUSSION}

Skin and soft tissue infections are one of the most common infectious diseases worldwide. ${ }^{16}$ The treatment of serious SSTIs require prompt anticipation of the severity of infection and timely debridement along with antibiotic cover for the control of infection. Though methicillin resistant Staphylococcus aureus (MRSA) remains one of the most common pathogens responsible for SSTIs but the etiological spectrum is changing, and now multidrug resistant Gramnegative organisms and vancomycin resistant enterococcus are amongst few emerging organisms from SSTIs. In the present study $4.63 \%$ of the samples were culture positive for streptococcal isolates. The most common was Enterococcus species (83.66\%)followed by Streptococcus pyogenes (7.84\%), Streptococcus agalactiae (6.53\%) and Streptococcus pneumoniae (1.96\%).The drug resistant Enterococcus species has emerged as an important nosocomial pathogen over past few years. In our study, $63.4 \%$ of Enterococcal isolates were resistant to Ampicillin while $70.3 \%$ showed resistance against Erythromycin, $44.3 \%$ were resistant against high-level Gentamicin (HLG). Vancomycin resistant Enterococcal isolates were $7.5 \%$. No resistance was found against linezolid. Such high level of drug resistance to multiple antibiotics in Enterococcal isolates have been observed by other authors also.17,18 Recent studies have shown that the vancomycin resistance can vary between 1.7$20 \%$ in the tertiary care hospitals of India as was seen in the present study also. ${ }^{19-21}$

In this present study, we have also investigated antimicrobial activity against $\mathrm{S}$. pyogenes isolates. Resistance was seen to the commonly used antimicrobials such as Erythromycin, Gentamicin (33.3\%). No resistance was seen against linezolid and vancomycin. Takashi Takahashi et al has recently reported a high frequency of resistance to erythromycin in Streptococcus pyogenes, particularly in countries where antibiotics are overused. ${ }^{22}$ Tamayo et al reported the erythromycin resistance rate to be $21.7 \%$ in the study done in Spain in 2004. ${ }^{23}$ Alberti et al had also reported a very high level of resistance to fluoroquinolones to the tune of $63.3 \%$ amongst S. pyogenes isolates. ${ }^{24}$ The increase in resistance in Streptococcus pyogenes isolates is a cause of worry as the SSTI caused by it can progress rapidly and may lead to necrotizing fasciitis and other sequelae if not treated promptly. This becomes important in patients who are allergic to penicillins where these alternate drugs would need to be used.

Streptococcus agalactiae is a major pathogen in neonatal infections. However, an increasing number of diseases caused by GBS in non-pregnant adult-invasive strains has been observed. 25, 26. In adults, S. agalactiae causes skin and soft tissue infections, bacteraemia, urinary tract infections, pneumonia, osteomyelitis, meningitis, endocarditis, and streptococcal toxic shock syndrome.25, 26 Risk factors associated with invasive GBS in adults are old age, diabetes mellitus, neurologic diseases, cirrhosis or other liver diseases, stroke, breast cancer, and renal failure. ${ }^{27-29}$ In our study, ten isolates of Streptococcus agalactiae and three isolates of Streptococcus pneumonia were recovered; considered as rare pathogens in suppurative infection. The increase in the prevalence of these relatively uncommon isolates and rising prevalence of drug resistance from the SSTIs warrants that culture and sensitivity should be done from all the suspected cases.

\section{CONCLUSIONS}

Streptococcus species such as S. pyogenes, S. Agalactiae and Streptococcus pneumoniae are seen to be increasing in SSTIs. Bacterial isolates exhibited high to moderate levels of resistance against different classes of antibiotics. The susceptibility data from this report may be worth consideration while implementing empiric treatment strategies for pyogenic infections. A continuous inspection should be carried out to monitor the susceptibility of these pathogens and chose appropriate regimens both for prophylaxis and treatment of surgical wound infections. There is a need to create a viable antibiotic policy and draft guidelines to prevent or reduce undirected use of antibiotics, and conserve their effectiveness for better patient management. Consistent dialogue between the microbiology department and the surgeons is required for management of such cases.

\section{REFERENCES}

[1] Euzéby JP. List of prokaryotic names with standing in nomenclature-genus Streptococcus. 2016.

[2] Facklam R. What happened to the Streptococci: overview of taxonomic and nomenclature changes. J Clin Microbiol 2002;15(4):613-30.

[3] Ferretti JJ, Stevens DL, Fischetti VA. Streptococcus pyogenes: basic biology to clinical manifestations. Oklahoma City (OK): University of Oklahoma Health Sciences Center: 2016.

[4] Lamagni TL, Efstratiou A, Vuopio-Varkila J, et al. The epidemiology of severe Streptococcus pyogenes associated disease in Europe. Euro Surveill 2005;10(9):179-84.

[5] Lancefield RC, Hare R. The serological differentia-tion of pathogenic and non-pathogenic strains of hemolytic Streptococci from parturient women. J Exp Med 1935;61(3):335-49.

[6] Badri MS, Zawaneh S, Cruz AC, et al. Rectal colonization with group B Streptococcus: relation to vaginal colonization of pregnant women. J Infect Dis 1977;135(2):308-12.

[7] Van der Mee-Marquet N, Fourny L, Arnault L, et al. Molecular characterization of human-colonizing Streptococcus agalactiae strains isolated from throat, skin, anal margin and genital body sites. J Clin Microbiol 2008;46(9):2906-11.

[8] Pandya N, Accardi KZ, Israelite C. Septic arthritis of the hip following group B psoas abscess in a postpartum patient resulting in total Hip arthroplasty. The Internet Journal of Orthopedic Surgery 2006;6(2):1-6.

[9] Ruppen C, Notter J, Strahm C, et al. Osteoarticular and skin and soft-tissue infections caused by Streptococcus agalactiae in elderly patients are frequently associated with bacteremia. Diagn Microbiol Infect Dis 2018;90(1):55-7. 
[10] Garcia-Lechuz JM, Cuevas O, Castellares C, et al Streptococcus pneumoniae skin and soft tissue infections: characterization of causative strains and clinical illness. Eur J Clin Microbiol Infect Dis 2007;26(4):247-53.

[11] Rajkumari N, Mathur P, Misra MC. Soft tissue and wound infections due to enterococcus spp. among hospitalized trauma patients in a developing country. Journal of Global Infectious Diseases 2014;6(4):189-93.

[12] Siesing PC, Alva-Jørgensen JP, Brodersen J, et al. Rising incidence of Enterococcus species in microbiological specimens from orthopedic patients correlates to increased use of cefuroxime: a study concentrating on tissue samples. Acta Orthop 2013;84(3):319-22.

[13] Wong SSY, Yuen KY. Streptococcus pyogenes and reemergence of scarlet fever as a public health problem. Emerging Microbes \& Infections 2012;1(7):e2.

[14] Rubinstein E, Keynan Y. Vancomycin-resistant enterococci. Crit Care Clin 2013;29(4):841-52.

[15] Clinical and Laboratory Standards Institute M100-S21 (2017). Performance standards for antimicrobial susceptibility testing. Twenty-first informational supplement. Wayne, PA: CLSI 2017;31:1.

[16] Guillamet CV, Kollef MH. How to stratify patients at risk for resistant bugs in skin and soft tissue infections? Curr Opin Infect Dis 2016;29(2):116-23.

[17] Thapa B, Tattawasart U, Manjai A, et al. The antimicrobial resistance and the species prevalence of the Enterococcal isolates in the Srinagarind Hospital, Northeastern Thailand. KKU Res J (GS) 2007;7(4):97108.

[18] Jain S, Kumar A, Kashyap B, et al. The clinicoepidemiological profile and the high-level aminoglycoside resistance in enterococcal septicemia at a tertiary care hospital in east Delhi. Int J App Basic Med Res 2011;1(2):80-3.

[19] Mulla S, Patel KG, Panwala T, et al. The prevalence of Enterococci with a higher resistance level in a tertiary care hospital: a matter of concern. National J Med Res 2012;2(1):25-7.
[20] Vidyalakshmi P, Gopalakrishnan R, Ramasubramanian $\mathrm{V}$, et al. The clinical, epidemiological and the microbiological profiles of the patients with vancomycin-resistant Enterococci from a tertiary care hospital. J Global Infect Dis 2012;4(2):137-8.

[21] Chaudhary U, Shamma M, Yadav A. The antimicrobial susceptibility patterns of the common and the unusual Enterococcus species which were isolated from clinical specimens. J Infect Dis Antimicrob Agents 2007;24(2):55-62.

[22] Takahashi T, Arai K, Lee DH, et al. Epidemiological study of erythromycin-resistant streptococcus pyogenes from Korea and Japan by emm genotyping and multilocus sequence typing. Ann Lab Med 2016;36(1):9-14.

[23] Tadayoshi I, Akihito W, Yoshishige I, et al. Dissemination of the phage-associated novel superantigen gene speL in recent invasive and noninvasive Streptococcus pyogenes M3/T3 isolates in Japan. Infection and Immunity 2002;70(6):3227-33.

[24] Ikebe T, Murai N, Endo M, et al. Changing prevalent T serotypes and emm genotypes of Streptococcus pyogenes isolates from Streptococcal Toxic Shock-Like Syndrome (TSLS) patients in Japan. Epidemiol Infect 2003;130(3):569-72.

[25] Blancas D, Santin M, Olmo M, et al. Group B streptococcal disease in nonpregnant adults: incidence, clinical characteristics and outcome. Eur J Clin Microbiol Infect Dis 2004;23(3):168-73.

[26] Wilder-Smith E, Chow KM, Kay R, et al. Group B streptococcal meningitis in adults: recent increase in Southeast Asia. Aust N Z J Med 2000;30(4):462-5.

[27] Corvec S, Illiaquer M, Touchais S, et al. Clinical features of group B Streptococcus prosthetic joint infections and molecular characterization of isolates. J Clin Microbiol 2011;49(1):380-2.

[28] Edwards MS, Baker CJ. Group B streptococcal infections in elderly adults. Clin Infect Dis 2005;41(6):839-47.

[29] Skoff TH, Farley MM, Petit S, et al. Increasing burden of invasive group B streptococcal disease in nonpregnant adults, 1990-2007. Clin Infect Dis 2009;49(1):85-92. 